\title{
Pneumomediastinum after transbronchial cryobiopsy
}

\author{
Emanuela Barisione1, Rita Bianchi2, Roberto Fiocca², Mario Salio1 \\ 1 Pulmonology Division, IRCCS Ospedale Policlinico San Martino, Genoa \\ 2 Pathology Division, IRCCS Ospedale Policlinico San Martino, University of Genoa, Italy
}

\begin{abstract}
Pneumomediastinum is defined as the presence of air or gas within the mediastinum and it rarely complicates bronchoscopy. We report, to our best knowledge, the first case of pneumomediastinum following a transbronchial cryobiopsy (TBLC). TBLC is considered a safe procedure as compared with both transbronchial biopsy and surgical lung biopsy. Systematic reviews, metanalysis and a Pubmed research, revealed that in literature no pneumomediastinum has been mentioned after TBLC. We report this case for to make it known to interventional pulmonologists the possibility that a pneumomediastinum can follow a TBLC. In our case the spontaneous resolution in few days did not require any interventions.
\end{abstract}

\section{Introduction}

Pneumomediastinum is defined as the presence of air or gas within the mediastinum and it rarely complicates bronchoscopy. It is caused both from a lesion of the trachea or from an alveolar rupture. We report, to our best knowledge, the first case of pneumomediastinum following a transbronchial cryobiopsy.

Correspondig author: Emanuela Barisione, Padiglione 15 ex IST, Largo Rosanna Benzi 10, 16132 Genova, Italy.

Mobile +39.3286344366 - Fax +39.010 .5556785 .

E-mail: emanuela.barisione@hsanmartino.it

Key words: Transbronchial cryobiopsy; interstitial lung disease; pneumomediastinum.

Contributions: EB, MS, manuscript drafting, bibliography research; RB, $\mathrm{RF}$, cryobiopsy analysis.

Conflict of interest: The authors declare no conflict of interest regarding this work.

Received for publication: 15 January 2018

Accepted for publication: 7 June 2018

(C) Copyright E. Barisione et al., 2018

Tipografia PI-ME Editrice, Italy

Monaldi Archives for Chest Disease 2018; 88:909

doi: 10.4081/monaldi.2018.909

This article is distributed under the terms of the Creative Commons Attribution Noncommercial License (by-nc 4.0) which permits any noncommercial use, distribution, and reproduction in any medium, provided the original author(s) and source are credited.

\section{Case Report}

The case of a 75-years-old Caucasian man, ex-smoker, was discussed in our interstitial lung disease (ILD) multidisciplinary group. The patient reported dry cough for about 8 months and gradual increase dyspnea for a few months. Anamnesis showed an epilepsy in treatment with levetiracetam and hypothyroidism treated with levotiroxin. He had a BMI of 29 and thorax examination revealed bibasal crackles. Spyrometric data highlighted normal flow and volume but the carbon monoxide diffusion (DLC0) was slighted decreased (52\%). Pulmonary artery pressure was normal. The computed tomography (CT) scan revealed a bilateral basal interstitial thickening, with traction bronchiectasis, but without honeycombing. We excluded other causes of ILD. The patient had no domestic or occupational environmental exposures, no history of drug toxicities and immunological data were negative. In the presence of Usual Interstial Pneumoniae (UIP) possible pattern, we decided to perform transbronchial lung cryobiopsy (TBLC). The biopsies were obtained under fluoroscopic guidance using the flexible bronchoscope inserted through the rigid tube. During the procedure we performed a bronchoalveolar lavage (BAL) in the lingula, two transbronchial cryobiopsies in left B9 bronchus and two in left B10 bronchus.

After two hours from the procedure, the patient had dysphonia and subcutaneous emphysema. An X ray showed pneumomediastinum. A CT scan showed pneumomediastinum and mild neck subcutaneous emphysema (Figure 1). The patient remained haemodynamically stable, therefore we have chosen a conservative approach. Slowly the symptoms and signs improved in the next two days and finally disappeared so the patient was discharged.

On histology slides four samples were examined: three of them showed patchy fibrosis, few fibroblastic foci and partial architectural distortion with alveolar bronchiolization, favouring the diagnosis of probable UIP pattern [1]. The fourth sample was represented by a longitudinal section of a bronchial wall with cartilage (Figure 2). The multidisciplinary group, according to most recent official ATS/ERS/JRS/ALAT statement [2], evaluated the UIP radiological pattern and the histological features, and concluded for a diagnosis of idiopathic pulmonary fibrosis (IPF).

\section{Discussion}

Pneumomediastinum can recognize three different causes: spontaneous pneumomediastinum, mucosal disruption and soft tissue infection. Most of the time the cause of pneumomediastinum following an endoscopic procedure is the Macklin effect [3], or ventilation-induced barotrauma, which causes mucosal disruption and spreading of air to nearby tissues via the fascial planes connecting them. The advent of TBLC has occurred over previous decade, it might soon be integrated in the routine diagnostic pathways [4]. TBLC is considered a safe proce- 

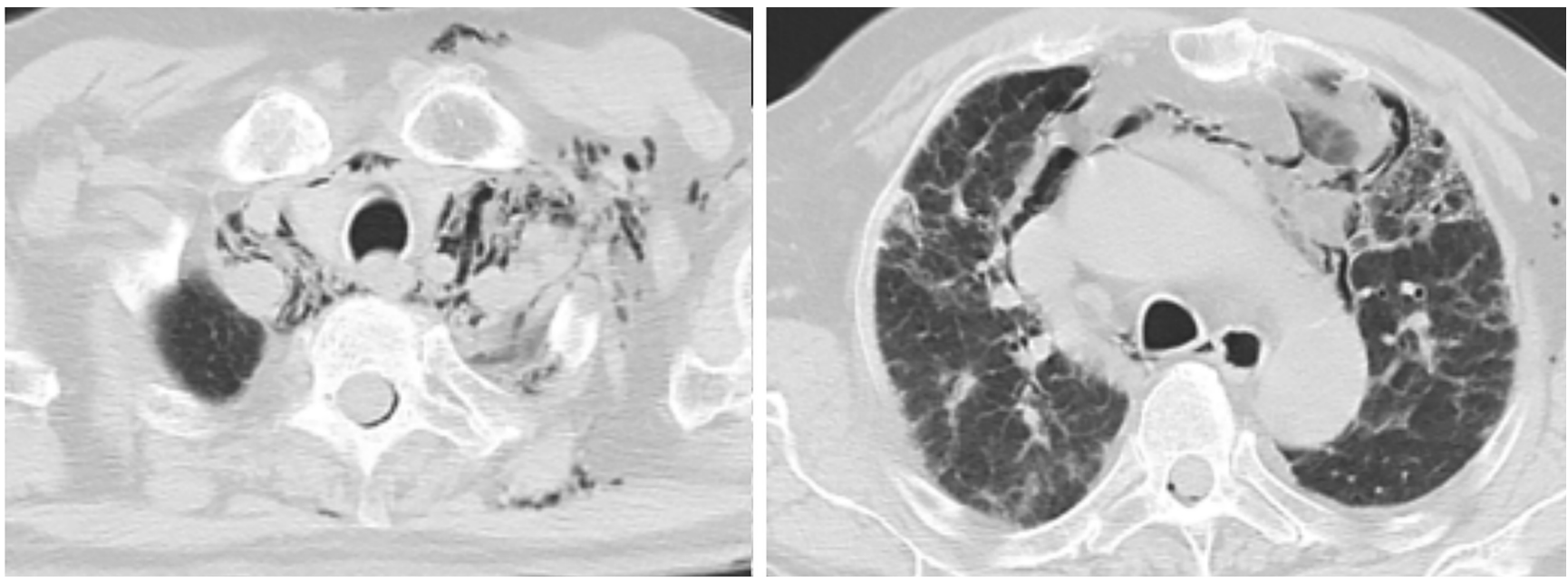

Figure 1. CT scan revealed air into mediastinum.

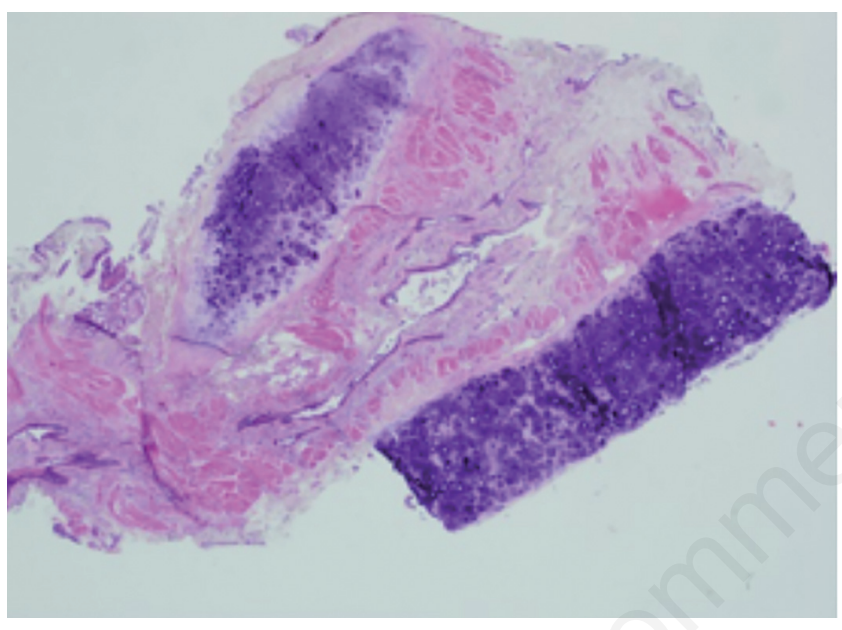

Figure 2. Longitudinal section of a bronchial wall with cartilage.

dure as compared with both transbronchial biopsy and surgical lung biopsy [5-8].

In a review published in 2016, focusing on the safety of transbronchial cryobiopsy, the most important adverse events of TBLC on 994 patients were pneumothorax and bleeding. Other signalled adverse events were prolonged air leak, transient respiratory failure, acute exacerbation, neurologic manifestation and one death [6]. Systematic reviews, metanalysis and a Pubmed research revealed that in the literature no pneumomediastinum has been mentioned after TBLC $[7,8]$.

In the present case we performed cryobiopsy using a rigid bronchoscope in a patient in deep sedation with propofol and remifentanil, without neuromuscular blockage, so we do not expect that the cause was barotrauma. One of the samples was taken too centrally and this induced air to penetrate into the mediastinum without causing pneumothorax.
We report this case for to make it known to interventional pulmonologists the possibility that a pneumomediastinum can follow a TBLC. In our case the spontaneous resolution in few days did not require any intervention.

\section{References}

1. Tabata K, Fukuoka J. Histopathologic features of usual interstitial pneumonia and related patterns: what is important for radiologists? Semin Ultrasound CT MR 2014;35:2-11.

2. Raghu G, Collard HR, Egan JJ. et al. An official ATS/ERS/JRS/ALALT statement: idiopathic pulmonary fibrosis: evidence-based guodelines for diagnosis and management. Am J Respir Crit Care Med 2011;183:788-824.

3. Maunder RJ, Pierson DJ, Hudson LD. Subcutaneus and mediastinal emphysema: pathophysiology, diagnosis, and management. Arch Intern Med 1984;144:1447-53.

4. Corte TJ, Collard H, Wells AU. Idiopatic interstitial pneumonias in 2015: a new era. Respirology 2015;20:697-8.

5. Gerhman E, Fruchter 0, Benjamin F, et al. Safety of cryotransbronchial biopsy in diffuse lung disease: analysis of three hundred cases. Respiration 2015;90:40-6.

6. Ravaglia C, Bonifazi M, Wells AU, et al. Safety and diagnostic yield of transbronchial lung cryobiopsy in diffuse parenchimal lung disease: a comparative study versus video assisted thoracoscopiclung biopsy and a systematic review of the literature. Respiration 2016;91:215-27.

7. Johannson KA, Marcoux VS, Ronksley PE, et al. Diagnostic yiel and complication of tranbronchial lung cryobiopsy for interstitial lung disease. A systematic review and meta-analysis. Ann Am Thorac Soc 2016;13:1828-38.

8. Iftikhar IH, Alghothani L, Sardi A, et al. Transbronchial lung cryobiopsy and video-assisted thoracoscopic lung biopsy in the diagnosis of diffuse parenchima lung disease. A meta-analysis of diagnostic test accuracy. Ann Am Thorac Soc 2017;14:1197-211. 